\title{
To go where no one has gone before: the necessity of radiobiology studies for exploration beyond the limits of the "Holy Gray" in radionuclide therapy
}

\author{
Frederik A. Verburg ${ }^{1}$ - Julie Nonnekens ${ }^{1,2,3} \cdot$ Mark W. Konijnenberg ${ }^{1,4} \cdot$ Marion de Jong ${ }^{1}$ \\ Published online: 4 January 2021 \\ (C) The Author(s), under exclusive licence to Springer-Verlag GmbH, DE part of Springer Nature 2021
}

Ever since the inception of nuclear medicine, dosimetry has been a topic of study and often a hot discussion. A number of editorials and position papers have been written in the European Journal of Nuclear Medicine and Molecular Imaging, detailing positions on the clinical use of dosimetry [1-4]. Moreover, the issue of whether to perform dosimetry has become politicized: The latest directives from the European Union surrounding this issue can be interpreted as both being very specific in mandating dosimetry or as a lack of a specific mandate for the same, depending on the precise interpretation of the legal phrasing — again providing the basis for the discussion within the nuclear medicine community. From different points of view, these aforementioned papers have detailed both the pros and cons, the good and the bad, as well as the superfluous and the sensible application of the array of dosimetric methods that have been and still are being developed as illustrated in, e.g. [5-11]. However, these past discussions have neglected to dive more deeply into potential avenues for exploration of the clinical relevance of dosimetry. Here, we will try and provide some ideas on possible ways forward in this respect.

The theoretical advantage of dosimetry, i.e. an individualized approach to radionuclide therapy aiming to prevent both overexposure of non-target organs and underexposure of

This article is part of the Topical Collection on Dosimetry

Frederik A. Verburg

f.verburg@erasmusmc.nl

1 Departments of Radiology and Nuclear Medicine, Erasmus MC, Dr. Molewaterplein 40, 3015 GD Rotterdam, Netherlands

2 Departments of Molecular Genetics, Dr. Molewaterplein 40, 3015 GD Rotterdam, Netherlands

3 Oncode Institute, Rotterdam, Netherlands

4 Department of Medical Imaging, Radboud UMC, Nijmegen, Netherlands target tissues, is clear and evident. However, thus far highlevel clinical proof that these advantages can be realized is largely lacking [3]. In fact, to some degree, some of the available evidence could even be interpreted to go against the usefulness of dosimetry. For instance, in the therapy of benign thyroid disorders - the oldest example of an in itself very successful radionuclide therapy - it has thus far repeatedly been shown that the total dose absorbed by the target tissue in some diseases is not related to the eventual success of treatment [12, 13]. In another example, in the more recently developed therapy with the prostate specific membrane antigen, the few studies available [14] thus far seem to indicate that the calculated delivered dose to cancer lesions has no more than a limited relationship with outcome. Some tumours can show a tremendous tracer uptake but fail to respond to radionuclide therapy, whereas other with at best modest uptake will show a great response - a clinical observation to which most of us who are involved in radionuclide therapy will be able to relate.

These examples clearly illustrate that dosimetry as practiced today is limited in its predictive power to relate radiation exposure to therapeutic response. The reasons for the limited predictive power may be manifold; however, the body of evidence in literature is thus far largely lacking in this respect. Nonetheless, from what is available and from biological theory, several main themes can be identified, which deserve further future exploration [15].

A first major potential cause of these differences in response is heterogeneity. This heterogeneity concerns both intra- and interpatient as well as physical factors. The intrapatient factors concern, e.g. the evolution of various different cancer cells, which could induce differences in radiation sensitivity between cancer cells. On top of that, even within the same metastasis, the uptake of radionuclides in different parts of tumour lesion could also vary from very high to completely absent [16]. This will of course lead to a differing response of cancer cells to therapy, as some will have received an excessive dose of radiation whereas others will have too little. In the 
case of radionuclide therapy, to some degree, cells that do not take up radionuclides may still be irradiated through uptake in neighbouring cells if such cell populations are intermixed and within the range of the radiation emitted. This effect may be less pronounced or even absent if different populations show regional clustering, let alone from separate metastases. Furthermore, this effect will be much less pronounced when using alpha-emitting radionuclides rather than beta-emitters. Currently, such effects are often not considered explicitly in clinical dosimetry - the dosimetric methods used (EUD or equivalent uniform dose) are based on external beam and brachytherapy, and only include extrinsic causes of nonuniform dose distributions - and it is unclear whether it plays a role in clinical practice. However, such effects may play a contributing role in determining the predictive power of the total lesion absorbed dose.

Heterogeneity in response to radiation can be intra-patient, where different lines of the same original tumour will develop additional genetic alterations, which may also result in an alteration of the response to genetic damage through ionizing radiation. It may however also concern inter-individual heterogeneity: The baseline response to the same radiation absorbed dose to the target may differ widely between individual patients. Whereas an absorbed dose of 300 Gy will lead to a complete ablation of the thyroid gland in some patients with Graves' disease, it is not even sufficient to cease hyperthyroidism in others [13] — while yet further patients showed a whole range of variants in response between these two extremes.

To complicate matters even further, no two cancers are the same. Even if cancers can be grouped by means of histopathology, in reality it concerns individual, highly variable diseases that also show significant degrees of variation on a genetic level.

Lastly, also not to be forgotten are the so-called abscopal and bystander effects, which describe effects only indirectly related to radiation exposure such as an activation of the immune system to non-irradiated foci in response to damage incurred by irradiated foci. These effects show both a high degree of heterogeneity and still are poorly understood [17].

Therefore, the heterogeneity in response to radiation exposures is a compounded product of all the previously mentioned inter- and intratumoral as well as intra- and interindividual differences, which is not taken into account in the physical absorbed dose.

Although such observations are hardly new, the scientific developments in this field have been not yet gained the prominence in the research agenda of most institutions they would deserve in light of the importance of these subjects with regard to understanding and improving radionuclide therapy. Largely unexplored remain a number of dosimetric parameters which may possibly be of influence. For instance, in a study by Krohn et al. [13], it was shown that not the total thyroid absorbed dose was related to therapeutic success of thyroid ablation in Graves' disease but rather the maximum dose rate to which the thyroid was exposed. Possibly, this might be explained by a high dose rate exceeding the cells' repair capacity, thus leading to cell kills, whereas a low dose rate, even at equivalent total absorbed dose, will be almost ignored in terms of cells' death when the cells' repair capacity is sufficient to undo any damage done by radionuclide therapy. As it is, this observation is valid for Graves' disease only; in other diseases, a similar phenomenon has not yet been describedin fact, in some diseases with, e.g., proliferative activity, the opposite may hold true. Rather a high total dose at lower dose rate may be more successful if over time it manages to catch the proliferative phase of more cells.

As a result, we certainly do not know nearly enough to understand this high variability. Research in radiobiology, i.e., the branch of science which explore the effects of ionizing radiation at cellular and subcellular level, including genomics and transcriptomics [18, 19], has for far too long received far too little attention [15] and, consequently perhaps, funding. Since the publication of some of the first dosimetric concepts by Marinelli et al. or Benua and Leeper [20, 21], many different diagnostic and therapeutic tracers have been developed, and the number of different alpha- and beta-emitting radionuclides available to nuclear medicine has expanded greatly. Yet we still in essence perform the same measurements based on the same premises as we did in times when the structure of DNA itself was not yet elucidated (1953). It is therefore that we explicitly call on the nuclear medicine community at large to consider this situation when discussing dosimetry and to increase the efforts to provide more clarity on the actions of radiation at a genetic level in order to provide a better understanding of what our target should be when performing dosimetry. Is it a total absorbed dose or a maximum dose rate? Is it a certain number of DNA double strand breaks (DSB) induced per MBq? Or is it another, yet unknown factor? Perhaps, in an ideal world, it will someday be possible to transpose the concept now used in the biologically effective and equivalent uniform dose to a deeper level and with a more profound understanding of the various factors involved in the heterogeneous response to radionuclide therapy or even radiation in more general terms to define a "genetically effective dose".

We should, of course, continue performing the studies on lesion dosimetry, blood dosimetry, bone marrow dosimetry, non-target organ dosimetry such as kidney dosimetry, etc. Although we could go on discussing whether there is a clinical sense and use for dosimetry as performed over the past $60+$ years, alternatively, we could try to dedicate our energy towards solving the great complex puzzle of the interaction between radiation and biology. The latter may one day provide answers, which will help those who all this is about: our patients. 


\section{Compliance with ethical standards}

Conflict of interest The authors declare that they have no conflict of interest.

Informed consent Not applicable.

Ethical approval The Institutional Review Board Approval was not required because the paper is an editorial.

\section{References}

1. Chiesa C, Sjogreen Gleisner K, Flux G, Gear J, Walrand S, Bacher $\mathrm{K}$, et al. The conflict between treatment optimization and registration of radiopharmaceuticals with fixed activity posology in oncological nuclear medicine therapy. Eur J Nucl Med Mol Imaging. 2017;44:1783-6.

2. Flux GD, Sjogreen Gleisner K, Chiesa C, Lassmann M, Chouin N, Gear J, et al. From fixed activities to personalized treatments in radionuclide therapy: lost in translation? Eur J Nucl Med Mol Imaging. 2018;45:152-4.

3. Strigari L, Konijnenberg M, Chiesa C, Bardies M, Du Y, Gleisner $\mathrm{KS}$, et al. The evidence base for the use of internal dosimetry in the clinical practice of molecular radiotherapy. Eur J Nucl Med Mol Imaging. 2014;41:1976-88.

4. Giammarile F, Muylle K, Delgado Bolton R, Kunikowska J, Haberkorn U, Oyen W. Dosimetry in clinical radionuclide therapy: the devil is in the detail. Eur J Nucl Med Mol Imaging. 2017;44: 2137-9.

5. Hanscheid H, Canzi C, Eschner W, Flux G, Luster M, Strigari L, et al. EANM Dosimetry Committee series on standard operational procedures for pre-therapeutic dosimetry II. Dosimetry prior to radioiodine therapy of benign thyroid diseases. Eur J Nucl Med Mol Imaging. 2013;40:1126-34.

6. Lassmann M, Haenscheid H, Chiesa C, Hindorf C, Flux G, Luster M. EANM dosimetry committee series on standard operational procedures for pre-therapeutic dosimetry I: blood and bone marrow dosimetry in differentiated thyroid cancer therapy. Eur J Nucl Med Mol Imaging. 2008;35:1405-12.

7. Hindorf C, Chittenden S, Aksnes AK, Parker C, Flux GD. Quantitative imaging of 223Ra-chloride (Alpharadin) for targeted alpha-emitting radionuclide therapy of bone metastases. Nucl Med Commun. 2012;33:726-32.

8. Hindorf C, Chittenden S, Causer L, Lewington VJ, Macke HR, Flux GD. Dosimetry for (90)Y-DOTATOC therapies in patients with neuroendocrine tumors. Cancer Biother Radiopharm. 2007;22:130-5.

9. Hindorf C, Emfietzoglou D, Linden O, Bousis C, Fotopoulos A, Kostarelos K, et al. Single-cell dosimetry for radioimmunotherapy of B-cell lymphoma patients with special reference to leukemic spread. Cancer Biother Radiopharm. 2007;22:357-66.
10. Hindorf C, Glatting G, Chiesa C, Linden O, Flux G, Committee ED. EANM Dosimetry Committee guidelines for bone marrow and whole-body dosimetry. Eur J Nucl Med Mol Imaging. 2010;37: $1238-50$.

11. Ljungberg M, Celler A, Konijnenberg MW, Eckerman KF, Dewaraja YK, Sjogreen-Gleisner K, et al. MIRD pamphlet no. 26: joint EANM/MIRD guidelines for quantitative 177Lu SPECT applied for dosimetry of radiopharmaceutical therapy. J Nucl Med. 2016;57:151-62.

12. Allahabadia A, Daykin J, Holder RL, Sheppard MC, Gough SC, Franklyn JA. Age and gender predict the outcome of treatment for Graves' hyperthyroidism. J Clin Endocrinol Metab. 2000;85:103842.

13. Krohn T, Hanscheid H, Muller B, Behrendt FF, Heinzel A, Mottaghy FM, et al. The maximum dose rate is a determinant of hypothyroidism after I-131 therapy of Graves' disease but the total thyroid absorbed dose is not. J Clin Endocrinol Metab. 2014;99: 4109 jc.2014-1347.

14. Violet J, Jackson P, Ferdinandus J, Sandhu S, Akhurst T, Iravani A, et al. Dosimetry of (177)Lu-PSMA-617 in metastatic castrationresistant prostate cancer: correlations between pretherapeutic imaging and whole-body tumor dosimetry with treatment outcomes. J Nucl Med. 2019;60:517-23.

15. Terry SYA, Nonnekens J, Aerts A, Baatout S, de Jong M, Cornelissen B, et al. Call to arms: need for radiobiology in molecular radionuclide therapy. Eur J Nucl Med Mol Imaging. 2019;46: 1588-90.

16. Sgouros G, Kolbert KS, Sheikh A, Pentlow KS, Mun EF, Barth A, et al. Patient-specific dosimetry for 131I thyroid cancer therapy using 124I PET and 3-dimensional-internal dosimetry (3D-ID) software. J Nucl Med. 2004;45:1366-72.

17. Widel M. Radionuclides in radiation-induced bystander effect; may it share in radionuclide therapy? Neoplasma. 2017;64:641-54.

18. Langen B, Rudqvist N, Spetz J, Helou K, Forssell-Aronsson E. Deconvolution of expression microarray data reveals 131Iinduced responses otherwise undetected in thyroid tissue. PLoS One. 2018;13:e0197911.

19. Spetz J, Rudqvist N, Langen B, Parris TZ, Dalmo J, Schuler E, et al. Time-dependent transcriptional response of GOT1 human small intestine neuroendocrine tumor after $(177) \mathrm{Lu}[\mathrm{Lu}]$-octreotate therapy. Nucl Med Biol. 2018;60:11-8.

20. Marinelli LD, Quimby EH, Hine G. Dosimetry of radioactive isotopes; biological observations and practical applications. Strahlentherapie. 1950;81:587-94.

21. Benua RS, Leeper RD. A method and rationale for treating metastatic thyroid carcinoma with the largest safe dose of I-131. In: Medeiros-Neto G, Gaitan G, editors. Frontiers in Thyroidology. 2nd ed. New York: Plenum Medical Book Co; 1986. p. 1317-21.

Publisher's note Springer Nature remains neutral with regard to jurisdictional claims in published maps and institutional affiliations. 